\title{
Funktionen als akteursabhängige Zuschreibungen in der Programmplanung betrieblicher Weiterbildung
}

\author{
Aiga von Hippel · Tina Röbel
}

Eingegangen: 5. November 2015 / Angenommen: 25. April 2016 / Online publiziert: 13. Mai 2016

(C) The Author(s) 2016. This article is available at SpringerLink with Open Access

Zusammenfassung Vorgestellt werden Ergebnisse eines DFG-Projekts zu Programmplanung betrieblicher Weiterbildung, das zwischen Juni 2013 und Mai 2015 am Lehrstuhl für Erwachsenenbildung/Weiterbildung an der Humboldt-Universität zu Berlin durchgeführt wurde. Die zentralen Fragestellungen lauteten: (1) Welcher Einfluss geht von den unterschiedlichen Akteurinnen und Akteuren auf die Programm- und Angebotsgestaltung (anvisierte Zielgruppen, Ziele, Inhalte, didaktische Handlungsfelder) in der betrieblichen Weiterbildung aus und (2) Welche spezifischen Formen der Programmplanung (u.a. in Bezug auf Handlungskoordination) erbringen welche Angebote und Programme? Das Forschungsdesign umfasste drei Unternehmensfallstudien, wobei in jedem Unternehmen neun bis zwölf Expertenund problemzentrierte Interviews sowie eine Programmanalyse durchgeführt wurden. Während es eine vergleichsweise hohe Erkenntnisdichte zur betrieblichen Weiterbildung in Bezug auf Einflussfaktoren auf Teilnahme und Strukturen gibt, lagen bislang nur wenige Studien spezifisch zur Programmplanung der betrieblichen Weiterbildung vor. Das Projekt leistete hierzu einen theoretisch-empirischen Beitrag. Als zentrales Ergebnis wird Programmplanung anhand der neu entwickelten Kategorie der von differenten Akteurinnen und Akteuren zugeschriebenen Funktionen betrieblicher Weiterbildung beschrieben. Ein weiteres wichtiges Ergebnis ist die Ausdifferenzierung unterschiedlicher Programmarten betrieblicher Weiterbildung.

Schlüsselwörter Betriebliche Weiterbildung · Programmplanung · Zugeschriebene Funktionen · Akteurinnen und Akteure betrieblicher Weiterbildung

Prof. Dr. A. von Hippel $(\varangle) \cdot$ T. Röbel

Humboldt-Universität zu Berlin, Berlin, Deutschland

E-Mail: aiga.von.hippel@hu-berlin.de

T. Röbel

E-Mail: tina.roebel@hu-berlin.de 


\title{
Functions as stakeholder-dependent ascriptions in program planning in the context of workplace learning
}

\begin{abstract}
The article presents the results of a DFG project on program planning of workplace learning, which was conducted between June 2013 and May 2015 at the Department of Adult Education/Continuing Education at the Humboldt University of Berlin. The central questions were: a) How do internal and external stakeholders influence the program planning and training design in the context of workplace learning (targeted audiences, objectives, contents, didactic fields of action)? b) Which specific forms of management and coordination of action deliver what kinds of results and benefits? The research design consisted of three company case studies. For each case study nine to twelve expert and problem-centered interviews, as well as a program analysis were conducted. So far the research on workplace learning has mainly offered insights on influencing factors of participation and organisational structures. There has been only a limited number of studies focusing specifically on program planning in the context of workplace learning. The contribution of the project is both empirical and theoretical. As a central result program planning is described with the help of a new category: the functions different stakeholders ascribe to workplace learning. Another important result is the detailed description of different program areas within workplace learning.
\end{abstract}

Keywords Workplace learning · Program planning · Ascribed functions · Stakeholders

\section{Einleitung und Vorstellung der Forschungsfragen und Forschungsziele}

Das Forschungsprojekt StePrA (Steuerung der Programm- und Angebotsplanung in der betrieblichen Weiterbildung. Eine Analyse der Handlungskoordination verschiedener Akteursgruppen $)^{1}$ untersuchte die Handlungskoordination unterschiedlicher Akteurinnen und Akteure bei der Programm- und Angebotsplanung in der betrieblichen Weiterbildung (bWB), die durch das Aushandeln von Macht und Interessen geprägt ist. Die unterschiedlichen außer- und innerbetrieblichen Akteurinnen und Akteure wirken steuernd auf die Programm- und Angebotsplanung ein. Innerhalb der bWB sind Personen mit unterschiedlichen beruflichen Hintergründen und damit heterogenen Wert- und Zielvorstellungen tätig. Die Zusammenarbeit der verschiedenen Akteurinnen und Akteure ist zentral für ein Gelingen der betrieblichen Bildungsarbeit. Es besteht die Notwendigkeit, sich abzustimmen, um unter Interdependenzbedingungen gemeinsam WB als Leistung zu erstellen.

Im Projekt wurde zwei zentralen Fragestellungen nachgegangen: (1) Welcher Einfluss geht von den unterschiedlichen Akteurinnen und Akteuren auf die Programmund Angebotsgestaltung (anvisierte Zielgruppen, Ziele, Inhalte, didaktische Handlungsfelder) in der bWB aus und (2) Welche spezifischen Formen der Programm-

\footnotetext{
${ }^{1}$ Das Projekt wurde von der DFG unter dem Kennzeichen HI 1599/1-1 gefördert.
} 
planung (u. a. in Bezug auf Handlungskoordination) erbringen welche Angebote und Programme? In einem ersten Schritt wurden somit Akteurinnen und Akteure, ihre Handlungskoordination bei der Programmplanung (Programme und Angebote) beschrieben, um diese in einem zweiten Schritt typisierend zu analysieren. Die Zusammenhänge wurden im Rahmen einer Grounded Theory verdichtet.

\section{Theoretischer Rahmen, Forschungslage und Entwicklung der Fragestellungen}

Die Theoriebezüge des Projekts liegen vorrangig in den erwachsenenpädagogischen Ansätzen zur Beschreibung und Analyse von Programmplanung (vgl. Gieseke 2003; Schlutz 2006; Dollhausen 2008; Cervero und Wilson 1994; von Hippel 2011, 2012). Zum anderen wurden auch betriebswirtschaftliche Ansätze berücksichtigt (vgl. stellvertretend Stender 2009; Becker 2011; sowie zur Unterscheidung der beiden Richtungen Käpplinger i. E.).

WB-Planungsprozesse verlaufen oftmals nicht nach einem konventionellen, zirkulären Planungsschema, sondern sind komplex und zum Teil konflikthaft aufgrund der Interessen unterschiedlicher Akteurinnen und Akteure. Dies zeigt die internationale wie die deutsche Literatur (z. B. zu Einflussgrößen auf kollektive bWBEntscheidungen Weber et al. 1994; Neuberger 1994; Käpplinger 2009).

Neben der grundsätzlichen Verortung des Forschungsvorhabens in der erwachsenenpädagogischen Programmforschung wurde die Governance-Perspektive genutzt, um den Blick auf Handlungskoordination der unterschiedlichen Akteurinnen und Akteure mit ihren unterschiedlichen Sinnlogiken, Verfügungsrechten und Zuschreibungen an bWB zu richten (vgl. Altrichter et al. 2007).

Im bisherigen Theorie- und Forschungsstand finden sich einige Studien dazu, welche Faktoren auf die Gestaltung der bWB Einfluss nehmen, die eher die Strukturen und auch Kooperationen und Netzwerke von Unternehmen und WB-Anbietern betrachteten (vgl. Übersicht bei Schiersmann et al. 2001 und Bäumer 1999, S. 42), weniger jedoch die tatsächliche Gestaltung und Handlungskoordination der Programmplanung. ${ }^{2}$ Bei diesen Studien wird deutlich (vgl. u. a. Weber et al. 1994), dass trotz vergleichbarer Strukturen unterschiedliche Entscheidungen im WB-Management in den Unternehmen getroffen werden können, so dass von einem - zwar durch Strukturen und Ressourcen als Rahmenbedingungen begrenzten - Gestaltungsspielraum ausgegangen werden kann (vgl. Bäumer 1999, S. 67). Heuer (2010) untersuchte die Aushandlungsprozesse bei WB-Entscheidungen. An diese Ergebnisse wurde im Projekt angeschlossen.

BWB wird als beigeordnete Bildung verstanden (vgl. Gieseke und Heuer 2011), da es nicht der primäre Zweck von Unternehmen ist, WB anzubieten (auch ,,se-

\footnotetext{
2 Für andere Aspekte bWB besteht eine vergleichsweise hohe Erkenntnisdichte. So z. B. zu Teilnahmequoten, Lernformen, Einflussfaktoren (wie z. B. Unternehmensgröße) auf Teilnahme an bWB sowie Strukturen (vgl. u. a. Bäumer 1999; Weber et al. 1994; Nienhüser und Rodehuth 1995; Heuer 2010; Schönfeld und Behringer 2013; BMBF 2014; Harney 1998; zur Konfigurationstheorie/bWB-Kulturen Käpplinger i. E.; zu Programmanalysen bWB-Angebote vgl. Pullig 1991).
} 
kundäre Erwachsenenbildungsinstitutionen“ genannt, vgl. Schmitz 1980, S. 121). Sie ist dadurch begründungspflichtig (vgl. Käpplinger i. E.) und in besonderem Maße kontingent, weil der Ressourceneinsatz für WB immer in Konkurrenz zu anderen Ausgaben steht. Das Programmplanungshandeln entsteht im Spannungsfeld von ökonomischen und pädagogischen Prinzipien. Das Spannungsfeld in der bWB eröffnet sich damit zwischen den Erwartungen, die an Wirtschaftlichkeit, kurzfristigem Erfolg und damit am Bedarf orientiert sind einerseits und Erwartungen, die an pädagogischen Zielen wie Kompetenzerwerb und Einsatz der Kompetenzen, langfristigem Nutzen und damit an den Bedürfnissen der Mitarbeitenden ausgerichtet sind, andererseits. Dabei deutet sich bislang keine Auflösung dieses Zielkonflikts an (vgl. Dobischat und Düsseldorff 2010). Umso wichtiger sind daher die der WB zugeschriebenen Funktionen. Dabei wurde bislang in der Literatur nur auf objektive Funktionen bWB abgehoben. Ein vielfacher Funktionsbezug ist implizit (vgl. zu Funktionen in anderen Weiterbildungsbereichen bspw. in der Qualifizierung und Fortbildung von Freiwilligen: Habeck 2015).

Die zentrale Kategorie der zugeschriebenen Funktionen in der Programmplanung bWB wurde im Verlauf des Projekts induktiv erarbeitet und anschließend am Theorie- und Forschungsstand gespiegelt (Details dazu im Unterpunkt 4.1.4). Der Schwerpunkt der vorgenommenen Analyse der zugeschriebenen Funktionen liegt dabei ebenfalls in der erwachsenenpädagogischen Programmforschung.

Der Begriff der Funktionen ist anschlussfähig an den bisherigen Theorie- und Forschungsstand. Dabei wurde bislang in der Literatur auf objektive Funktionen bWB abgehoben. Demgegenüber gehen wir dem interpretativen Paradigma folgend mit der Perspektive des Symbolischen Interaktionismus von einer subjektiven Wirklichkeitsund Bedeutungskonstruktion aus (vgl. Blumer 1972). Den Begriff der zugeschriebenen Funktionen verstehen wir dementsprechend als subjektive Bedeutungszuschreibung an bWB. Es wird angenommen, dass bWB nicht bestimmte Funktionen hat, sondern dass ihr vielmehr von den verschiedenen Akteuren unterschiedliche (auch mehrere) Funktionen zugeschrieben werden. Funktionen sind akteursabhängige Zuschreibungen in der Programmplanung bWB. Diese Zuschreibungen werden als eine Auslegung von Weiterbildung verstanden. ${ }^{3}$ Wir verwenden den Funktionsbegriff also nicht für eine soziologische Analyse beispielsweise der Funktionen bWB für gesellschaftliche (Teil-)Systeme. Dennoch ist bspw. die funktionale Analyse nach Merton (1967) anknüpfungsfähig, insofern als dass deutlich wird, dass Phänomene verschiedene Funktionen ausüben können und dass unterschiedliche soziale Gruppen Phänomene als unterschiedlich (dys-)funktional einschätzen können. Auch die

\footnotetext{
3 Wir haben uns für den Begriff der Funktionen entschieden, weil dieser anschlussfähig an die bisherige Literatur ist (,objektive“ Funktionen bWB). Den Begriff „Nutzen“ verwenden wir hier nicht; dieser verweist immer auf eine subjektive Bewertungsdimension in Bezug auf das Lernergebnis bzw. die Teilnahme (vgl. Fleige 2015, S. 190 - näher dran ist der Begriff der Nutzenzusammenhänge, vgl. ebd.). Zugeschriebene Funktionen können, müssen aber nicht auf Lernergebnisse zielen. Der Nutzen-Perspektive von Fleige und unserer gewählten Perspektive der zugeschriebenen Funktion ist gemeinsam der Fokus auf die Akteursperspektive (beim Nutzen der Fokus auf die Teilnehmenden, bei den zugeschriebenen Funktionen der Fokus auf die planenden Akteure).
} 
Grundfragen der funktionalen Analyse (Welche Folgen hat das? Wem nützt das?) sind für uns leitend. ${ }^{4}$

Hinsichtlich des Theorie- und Forschungsstandes bleibt festzuhalten, dass es bis auf einige Ausnahmen (u. a. Schmitz 1978, 1980; Harney 1998) noch wenige grundlegende theoretische Arbeiten zur Programmplanung bWB gibt (vgl. Käpplinger i. E.). Es lagen bislang weder empirische Detailanalysen zur Handlungskoordination auf der Mesoebene mit Fokus auf die Akteurinnen und Akteure, ihre unterschiedlichen Handlungslogiken und Aushandlungsprozesse (vgl. Schrader 2001; Käpplinger i. E.) vor, ${ }^{5}$ noch umfangreiche, mit Analysen zur Programmplanung verknüpfte Programmanalysen für die bWB. Als Forschungslücke war zu sehen, dass ,sich die Forschung zum Weiterbildungsmanagement (...) nicht auf die Akteure, sondern auf die Aufgaben und Methoden des Managements“ bezog (Schiersmann et al. 2001, S. 20).

\section{Methode und Design}

Es wurden drei explorativ-deskriptive (vgl. Fragestellung 1), explanativ typisierende (vgl. Fragestellung 2), vergleichende Betriebs-Fallstudien durchgeführt (vgl. Yin 2009). Diese umfassten neben Experteninterviews sowie problemzentrierten Einzelinterviews auch je eine Programmanalyse. Es kam ein vierstufiges qualitatives Triangulationsverfahren zum Einsatz (vgl. Flick 2004), das in Perspektivverschränkung ,eine Wirklichkeit aus den Schnittmengen der Perspektiven, die in kommunikative Wissens- und Handlungserarbeitung verwickelt sind“" (Gieseke 2007, S. 18), zeichnet. Durch dieses Design konnten nicht nur die Perspektiven der relevanten Akteurinnen und Akteure berücksichtigt, sondern auch der Einfluss auf das WBProgramm erfasst werden.

\subsection{Auswahl der Fälle}

Die Auswahl der Unternehmen ${ }^{6}$ erfolgte kategoriengeleitet nach dem Prinzip des theoretical sampling (vgl. Strauss und Corbin 1996). Damit wurde eine inhaltliche Repräsentation, aber keine statistische Repräsentativität (vgl. Merkens 1997) angestrebt. Als Grundgesamtheit wurden Unternehmen mit mindestens 2800 Mitarbeitenden gewählt. Nach Bäumer (1999) ist bei dieser Größe davon auszugehen, dass die Organisation die folgenden Kriterien erfüllt: Funktionsbereich WB ist vor-

\footnotetext{
${ }^{4}$ Funktionen nach Merton beziehen sich jedoch auf „beobachtbare objektive Konsequenzen“ (1967, S. 124) oder Folgen eines Phänomens. Merton bezeichnet wiederum Ziele, Motive und Zwecke als subjektive Dispositionen. Motive führen demnach zu Verhaltensweisen, ,aus denen sich (nicht notwendig intendierte) Funktionen ergeben können“ (ebd.). Nach dieser Terminologie könnte man sagen, dass wir von subjektiven Dispositionen der Akteure sprechen.

5 Analysen zu den unterschiedlichen Interessen von Arbeitnehmern und Arbeitgebern liegen bereits vor, jedoch nicht zu den ausdifferenzierten Akteuren, die an der Weiterbildungsplanung beteiligt sind.

${ }^{6}$ Die Auswahl der Unternehmen war wie erwartet sehr zeitaufwendig. Es wurden insgesamt Gespräche mit 15 Unternehmen geführt, von denen sich schließlich drei zur Kooperation bereit erklärt haben.
} 
handen, hauptamtliche WB-Stellen sind vorhanden, WB-Budget ist vorhanden, WB ist formal organisiert (vgl. Bäumer 1999, S. 221).

Um eine möglichst heterogen geschichtete Stichprobe zu erhalten, wurde ein weiteres Kriterium herangezogen: die WB-Aktivität der Branche. Es wurden drei unterschiedliche Branchen gewählt, von denen zwei statistisch betrachtet stark weiterbildungsaktiv sind (Fall A und C) und eine nur wenig (Fall B) (Schönfeld und Behringer 2013, S. 116 ff.).

\subsection{Durchführung der Interviews}

Nach einem Pretest fand in jedem der drei Unternehmen das erste Experteninterview mit der Leitung der für WB zuständigen Abteilung statt. Diese Person stellte den Kontakt zu den weiteren Interviewpartnern her: Führungskräfte verschiedener Hierarchieebenen, weitere Mitarbeitende der WB-Abteilung, Betriebsratsmitglieder und Mitarbeitende ohne Führungsverantwortung.

Mit Interviewpartnern, bei denen das Planen von WB-Angeboten zur Stellenbeschreibung gehörte, wurden Experteninterviews geführt (16 Interviews) (vgl. Bogner und Menz 2002). Wenn die Interviewpartner nur teilweise in die Planung von WBAngeboten oder lediglich in deren Nutzung involviert waren, wurden problemzentrierte Interviews geführt (19 Interviews) (vgl. Witzel 2000). Am Ende jeder Fallstudie wurde im Sinne einer kommunikativen Validierung ein abschließendes Experteninterview mit der WB-Leitung geführt (vgl. Mayring 2003). Insgesamt wurden 35 Interviews geführt.

\subsection{Auswertung}

Die Auswertung der Interviews (vollständig transkribiert, in MAXQDA kodiert) erfolgte in Anlehnung an die qualitative Inhaltsanalyse nach Mayring (2003) sowie nach den Kodierarten der Grounded Theory (vgl. Strauss und Corbin 1996). Um die intersubjektive Nachvollziehbarkeit zur überprüfen, wurden zwei Interviews von jeweils unterschiedlichen Personen parallel kodiert. Die Analyse der IntercoderReliabilität diente dem Ziel einer qualitativen Verbesserung des Kodierens und des Kodierleitfadens.

Der Programmanalyse lag in allen drei Fällen der gedruckte Programmkatalog zugrunde. Dieser umfasste die im Voraus durch die WB-Abteilung geplanten Angebote. Auf dieser Grundlage wurden organisationsbezogene, integrierte Programmanalysen durchgeführt (vgl. Robak 2012; Nolda 2009). Je Fall wurde dabei das WBProgramm eines gesamten Jahres betrachtet.

\section{Ausgewählte Ergebnisse}

Es handelt sich bei unserer Analyse um eine Analyse der Programmplanung bWB, bei der über zugeschriebene Funktionen die Programmplanung und das ErgebnisProgramm näher beschrieben werden können. Zentrale Ergebniserträge des Projekts liegen im Aufzeigen der unterschiedlichen beteiligten Akteurinnen und Akteure und 
ihren Funktionszuschreibungen an bWB sowie unterschiedlicher Programmarten. Zusammenfassend geht es um Folgendes:

Akteure (...), die sich letztlich über ihre Unterschiedlichkeit und ihre intentionalen Handlungen beschreiben lassen (...), beziehen sich in ihren Handlungen auf Strukturen und (re-)produzieren diese (...). Diese und die darauf fußenden Leistungen können als Ergebnisse der koordinierten Handlung aufgefasst und evaluiert werden (Altrichter und Heinrich 2007, S. 71 f.).

Die folgende Grafik zeigt anhand des Kodierparadigmas der Grounded Theory die grundlegenden Zusammenhänge. Diese werden anschließend zunächst zusammenfassend dargestellt (4.1) und dann anhand einer Fallanalyse (4.2) vertiefend erläutert.

\subsection{Programmplanung in der betrieblichen Weiterbildung: Akteuren zugeschriebene Funktionen und Programmarten}

Als zentrales Phänomen sehen wir die induktiv gewonnene Kategorie der akteursabhängig zugeschriebenen Funktionen an bWB (im Unterschied zur bisherigen Literatur, die hauptsächlich auf objektive Funktionen ohne Akteursunterschiede abhebt) (Abb. 1). Es ist das, worum es den befragten Akteurinnen und Akteuren hauptsächlich geht. Die Funktionen sind eingebettet in verschiedene Kontexte und Bedingungen. Die Strategien, wie die Akteurinnen und Akteure mit den zugeschriebenen Funktionen umgehen, zeigen sich in den Programmplanungsprozessen. Diese Programmplanungsprozesse kann man anhand verschiedener theoretischer Modelle klassifizieren und empirisch beschreiben. Als Resultat der auf die zugeschriebenen Funktionen bezogenen Programmplanungsprozesse lassen sich verschiedene Programmarten klassifizieren und empirisch beschreiben.

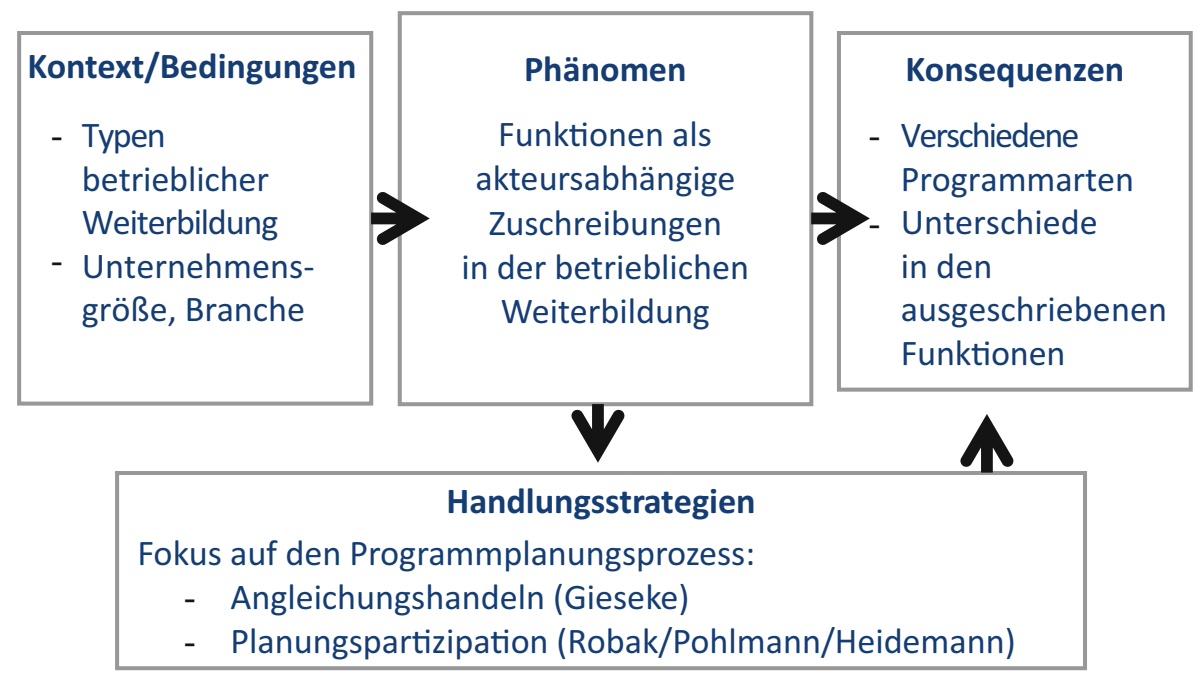

Abb. 1 Akteursabhängig zugeschriebene Funktionen als zentrales Phänomen (Kodierparadigma nach Strauss und Corbin 2006) 


\subsubsection{Kontext und Bedingungen}

Aus dem bisherigen Literatur- und Forschungsstand bekannt, gehören zu den Einflussfaktoren und Rahmenbedingungen bWB insbesondere Unternehmensgröße, Branche, Engagement in der Ausbildung, der Anteil der qualifizierten Beschäftigten, die Existenz eines Betriebsrats, das Innovationsniveau (vgl. Schönfeld und Behringer 2013), sowie die Typen betrieblicher Weiterbildungsorganisation (vgl. Bäumer 1999). Welche Funktionen bWB zugeschrieben werden, hängt darüber hinaus von den jeweiligen individuellen Akteurinnen und Akteuren ab (u. a. Aufbauorganisation, beruflicher Hintergrund), aber auch von Ressourcen, Unternehmensphilosophie, gesetzlichen Vorgaben.

Anstatt von individuellen Akteuren zu sprechen, könnte man die Weiterbildungsabteilung auch als korporativen und den Betriebsrat als kollektiven Akteur untersuchen. Wir sprechen jedoch von individuellen Akteuren, da deutlich wurde, dass die individuellen Akteure auch innerhalb eines korporativen Akteurs der bWB unterschiedliche Funktionen zuweisen können (vgl. zum Akteursbegriff in der Governance-Forschung Altrichter et al. 2007). ${ }^{7}$

Im Projekt wurden deutlich in allen drei Fallstudien die Akteurinnen und Akteure, die an der Planung bWB direkt oder indirekt beteiligt sind, ${ }^{8}$ herausgearbeitet: WB-Abteilung, Führungskräfte, interne und externe Trainer, Unternehmensleitung, Mitarbeitende, Betriebsrat. Als weitere Akteurinnen und Akteure bei nicht allen Unternehmen sind zu nennen: Unternehmensleitung, Personalleitende, Kunden des Unternehmens, Gesetzgeber, regionale Personalentwickler, zentrale Personalentwicklungs-Stabsstellen. Die Akteurinnen und Akteure haben jeweils eigene Sinnlogiken, die sich in den unterschiedlichen zugeschriebenen Funktionen widerspiegeln und die ausgehandelt werden müssen.

\subsubsection{Handlungsstrategien}

Es wird davon ausgegangen, dass Programmplanung so wie von Gieseke (2003) beschrieben als Angleichungshandeln verstanden werden kann und dass dabei Gestaltungsspielräume vorhanden sind - sowohl im Prozess des Angleichungshandelns als auch in Bezug auf das Programm als Ergebnis. Das Hauptinteresse des Projekts war es daher, zu verstehen, warum bestimmte Programme auf bestimmte Art und Weise gestaltet wurden (vgl. auch McLean 2000). Die Erschließung der Zuschreibungen der Akteurinnen und Akteure ermöglicht insbesondere auf die Frage des Warum Antworten zu geben. Über die akteursabhängig zugeschriebenen Funktionen kann

\footnotetext{
7 Obwohl in der Governance-Perspektive soziale - d.h. überindividuelle Akteure - fokussiert werden, sollte die Analyse der individuellen Akteure nicht vernachlässigt werden, da sie es sind, die mit ihren Problemwahrnehmungen und Deutungsmustern handeln (vgl. Altrichter und Heinrich 2007, S. 57 f.).

8 Vgl. zu quantitativen Berechnungen bzgl. der Akteure bei Weiterbildungsentscheidungen: An Weiterbildungsentscheidungen sind gerade in großen Unternehmen mehrere Akteure beteiligt. Hohen Einfluss auf Weiterbildungsentscheidungen in Unternehmen mit mehr als 500 Beschäftigten haben Vorgesetzte, gefolgt von der Personalabteilung, einzelnen Abteilungen, danach folgen Geschäftsführer und Mitarbeitende (vgl. Käpplinger 2012).
} 
Angleichungshandeln in der bWB als Auslegungsprozess mit unterschiedlichen Akteurinnen empirisch beschrieben werden.

Die Programmplanung als Handlungsstrategie wurde unter verschiedenen theoretischen Aspekten empirisch analysiert. So beispielsweise als Aushandeln von Macht und Interessen, über die die Akteurinnen und Akteure in unterschiedlichem Rahmen und unterschiedlicher Ausgestaltung verfügen (vgl. Cervero und Wilson 1994), unter dem Aspekt der Planungspartizipation (vgl. Robak et al. 2015) oder als Planungskultur (vgl. Dollhausen 2008).

\subsubsection{Konsequenzen}

Aus den unterschiedlichen zugeschriebenen Funktionen und Programmplanungsprozessen ergeben sich differente Programmarten. ${ }^{9}$ In allen drei Unternehmen gibt es eine Vielzahl an WB-Angeboten jenseits des jeweiligen Programmkatalogs. Diese unterschiedlichen Programmarten in der bWB waren bislang kaum systematisch und empirisch erfasst. ${ }^{10}$ Im Fallvergleich schlagen wir folgende Einordnung vor:

- Programmkatalog: die von der WB-Abteilung im Voraus geplanten, offenen Angebote,

- bereichsinterne Angebote: von verschiedenen Unternehmensbereichen/Gruppen/ Standorten im Unternehmen eigenständig geplante Angebote ohne Beteiligung der WB-Abteilung,

- externe Angebote: Angebote externer Anbieter, die für einzelne Mitarbeitende gebucht werden, entweder mit oder ohne Beteiligung der WB-Abteilung,

- maßgeschneiderte Angebote: Angebote, bei denen die WB-Abteilung als Dienstleister fungiert und kurzfristig auf Bedarf aus unterschiedlichen Unternehmensbereichen reagiert,

- weitere Einzelangebote: weitere WB-Angebote bzw. Aktivitäten der WB-Abteilung, wie z. B. Schulungsmaßnahmen im Rahmen einer Kampagne gegen sexuelle Gewalt.

Die Programmarten unterscheiden sich hinsichtlich der planenden Akteurinnen und Akteure, der jeweils zugeschriebenen Funktionen, der Zielgruppe, der Themen, der Finanzierung und des Planungshorizonts. In den jeweiligen WB-Angeboten spiegeln sich unterschiedliche funktionale Bezüge.

\footnotetext{
9 Da der Begriff „Programmbereich“ konnotiert ist mit den unterschiedlichen Fachbereichen u. a. an VHS, sprechen wir bei der Ergebnisdarstellung von „Programmarten“, um die unterschiedlichen Programmsegmente und -formen deutlich zu machen.

10 Pullig (1991) zeigt zwar bereits auf, dass in vielen Unternehmen neben den „Standardprogrammen“ bedarfsorientierte Angebote geplant werden, allerdings wird in dem Beitrag keine systematische Differenzierung der unterschiedlichen Angebotsbereiche vorgenommen. Gleichzeitig weist er darauf hin, dass Programmanalysen in der bWB vor der Schwierigkeit stehen, dass nicht für alle Angebote gedruckte Programme vorliegen.
} 


\subsubsection{Phänomen}

Wie bereits im Theorie- und Forschungsstand dargestellt, wird nicht davon ausgegangen, dass bWB bestimmte Funktionen innehat, sondern, dass ihr vielmehr unterschiedliche Funktionen zugeschrieben werden. ${ }^{11}$ Funktion wird definiert als der Zusammenhang, den die Akteurinnen und Akteure zwischen WB und anderen Zielen herstellen. Andere Ziele können sowohl Organisationsziele sein als auch sich auf die Interessen einzelner Akteurinnen und Akteure beziehen. Unterschiedliche Akteurinnen und Akteure weisen bWB bzw. den jeweiligen Programmarten unterschiedliche Funktionen zu. In diesem Sinne können die Funktionen als eine empirische Spezifizierung der beigeordneten Bildung im betrieblichen Kontext verstanden werden. Gleichzeitig gehen wir davon aus, dass sich auch der Zeitgeist in den Funktionen widerspiegelt, und bspw. einige der Funktionen einer neoliberalen Haltung zugeordnet werden können.

Die grundlegenden Arbeiten zu objektiven Funktionen bWB stammen von Schmitz (1978, 1980), Diedrich (1988) und Weber (1985). Auf diesen fußen die weiteren Arbeiten (vgl. Geißler und Orthey 1990; Harney 1998; Faulstich 1998; Rodehuth 1999; Quenzler 2008; Heuer 2010; Dewe und Feistel 2013; Seyda und Werner 2014). Es wird in der Regel systemtheoretisch fundiert auf drei Ziel-Perspektiven bWB verwiesen: Individuen bzw. Mitarbeitende, Unternehmen, Gesellschaft. ${ }^{12}$ Aus personalwirtschaftlicher Sicht hat bWB die Funktion, den Faktor Arbeit - mit dem Ziel der Produktivität des Unternehmens - bereitzustellen und zu sichern, zu problemadäquaten Kosten (vgl. Rodehuth 1999). Die in der Literatur genannten Funktionen spiegeln oft die personalwirtschaftlichen Grundfunktionen. Die verschiedenen Akteurinnen und Akteure und ihre jeweiligen Perspektiven werden nicht berücksichtigt, ebenso wenig dass unterschiedlichen Programmarten unterschiedliche Funktionen zugeschrieben werden.

Nach Schmitz liegen die Funktionen von bWB zwischen den ,Polen betrieblicher Systemprobleme, die Produktion von Gütern und Dienstleistungen effizient zu gestalten und die Entscheidungsmonopole der hierarchischen Spitze legitimatorisch zu sichern bzw. dementsprechend ein sowohl an Leistung als auch an Loyalität gemessenes Arbeitshandeln zu erreichen“ (Schmitz 1980, S. 123). Schmitz industriesoziologische Analyse unterscheidet vier personalpolitische Funktionen unter den Aspekten von gesellschaftlicher Arbeitsteilung und Herrschaft: (1) technisch-instrumentelle Fähigkeiten der Beschäftigten anzupassen; (2) Wissen an Führungskräfte zu vermitteln um die hierarchische Struktur aufrechtzuerhalten; (3) Disziplinierung und Motivierung der Beschäftigten für Aufstiege (vertikale Mobilität); (4) Gratifikation und Spaltung betrieblicher Arbeitsmärkte: geringe Qualifizierungskosten für Mitarbeiter, deren Arbeitsplätze bei Konjunkturschwankungen etc. eingespart werden und mehr Investitionen für die Stammbelegschaft. Übergreifend lässt sich sagen,

\footnotetext{
11 Vgl. Harney 1998: „Differenzen zwischen den verschiedenen Kontexten der Weiterbildung werden nicht auf der Ebene der Themen und Inhalte markiert. Markiert werden sie auf der Ebene der kommunikativen Rahmung, in die die Themen und Inhalte eingefaßt sind“ (ebd., S. 140).

12 Funktionen werden dabei als objektive Kategorien beobachtbarer Folgen gesehen, die von subjektiven Kategorien der Disposition unterschieden werden können (vgl. Merton 1967, S. 125).
} 
dass Schmitz neben der Qualifizierungsfunktion (1 und 2) insbesondere die Selektions- und Legitimationsfunktion/soziale Reproduktionsfunktion (3 und 4) als zentrale Funktionen herausarbeitet.

Demgegenüber enthält unser Verständnis der zugeschriebenen Funktionen keine Aussage darüber, inwiefern die Funktionen im jeweiligen Unternehmen/in Bezug auf die jeweilige Programmart erfüllt werden. Es geht nicht darum, objektiv Zusammenhänge zu unterstellen, sondern darum, über zugeschriebene Funktionen die Gestaltung der Programmplanung erklären zu können. Man könnte in gewisser Weise auch sagen, dass es sich um erwartete Funktionen handelt.

In der folgenden Tab. 1 werden alle induktiv erarbeiteten sowie deduktiv ergänzten Funktionen zusammengeführt. Sie werden danach unterschieden, inwiefern das Lernergebnis eine hohe oder eher eine niedrige Relevanz besitzt. ${ }^{13}$ Deduktiv meint hier, dass die Funktionen in der Literatur genannt werden, wenn sie dort auch als objektiv und nicht als zugeschrieben verstanden werden.

Einige der oben genannten Funktionen können auch erfüllt sein, ohne dass die WB-Angebote tatsächlich stattfinden. Um beispielsweise im Sinne der Akquisefunktion im Wettbewerb um gute Fachkräfte erfolgreich zu sein, ist es ausreichend, ein möglichst umfangreiches Weiterbildungsprogramm in Form eines Trainingskatalogs zu gestalten, um dieses bei Vorstellungsgesprächen auf den Tisch zu legen. ${ }^{14}$

Ebenso wichtig ist es zu reflektieren, welche Funktionen von den Akteuren nicht genannt werden. Gesellschaftliche und kulturelle Teilhabe bspw. werden von den Interviewpartnern nicht angesprochen. Wenig genannt werden auch die Entwicklungsfunktion oder die Unterstützung von Gesundheit, Wohlbefinden, Zufriedenheit ohne Bezug zur Arbeitsfähigkeit. Selbst bei Fall B, dem Unternehmen mit dem am stärksten erwachsenenpädagogisch geprägten Zugang zu bWB (Mitarbeitende verfügen über Studienabschlüsse in Erwachsenenbildung), liegt der Fokus auf der Abschaffung der organisierten Angebote. Das Ziel ist es, Lernen im Arbeitsprozess zu fördern und Selbstlernprozesse mit digitalen Medien zu unterstützen (ähnlich auch Fall A). Dabei wird nicht reflektiert, welche Mitarbeitergruppen an dieser Art des Lernens vermutlich nicht partizipieren können (bekannte Forschungsergebnisse).

Warum kaum Funktionen mit Bezug zu Teilhabe und Chancengerechtigkeit sowie Bildung als Selbstzweck angesprochen werden, lässt sich über die Handlungslogik bWB erklären. Harney (1998) arbeitet systemtheoretisch und auch bildungsökonomisch fundiert die Handlungslogik bWB heraus. Leitgesichtspunkt betriebli-

\footnotetext{
13 Eine weitere Möglichkeit wäre, zwischen individuellen und organisationalen Lernergebnissen zu unterscheiden. Letztere führen zu einem Reflexiv-Werden von Strukturen, also der Herausbildung von Strukturen zur Änderung der Strukturen bzw. der Entwicklung von Regeln zur Entwicklung von Regeln (vgl. Behrmann 2010, S. 94 f.). So können z. B. Bewältigungs- und Flexibilitätsfunktion auch Aspekte organisationalen Lernens enthalten.

14 Mit Merton könnte man hier von einer objektiven Konsequenz der subjektiven Disposition der Leitung der WB-Abteilung sprechen (vgl. 1967). Beispielsweise könnte man das Ziel der Leiterin der Personalabteilung in Fall A im Wettbewerb um gute Fachkräfte erfolgreich zu sein, als subjektive Disposition bezeichnen. Das führte zu der Verhaltensweise, ein möglichst umfangreiches Weiterbildungsprogramm in Form eines Trainingskatalogs zu gestalten. Beobachtbare objektive Konsequenz ist in diesem Fall, dass bei Vorstellungsgesprächen dieser Trainingskatalog auf den Tisch gelegt wird. Weiterführende Analysen könnten darüber hinaus neben dieser manifesten Funktion auch latente Funktionen, d. h. unerwartete und unerkannte, untersuchen.
} 
Tab. 1 Zugeschriebene Funktionen an betriebliche Weiterbildung

\begin{tabular}{lll}
\hline $\begin{array}{l}\text { Funktionen mit hoher Rele- } \\
\text { vanz der Lernergebnisse }\end{array}$ & Beschreibung & Quelle \\
\hline
\end{tabular}

1. Qualifizierungs- und Versorgungsfunktion

2. Bewältigungs- und Flexibilitätsfunktion

3. Integrations- und Steuerungsfunktion

4. Gesunderhaltungsfunktion

5. Entwicklungsfunktion

\section{Funktionen mit geringer Relevanz der Lernergeb- nisse}

6. Image- und Akquisefunktion

\section{Compliance-Funktion}

8. Gratifikations-, Motivations-, Mitarbeiterbindungsfunktion

9. Selektions- und Legitimationsfunktion, soziale Reproduktionsfunktion

10. Ablenkungsfunktion

11. Profilierungsfunktion: Selbstinszenierungs-und Einflussfunktion

12. Networking-Funktion
Versorgt das Unternehmen mit den notwendigen Qualifikationen/qualifizierten Mitarbeitenden, stellt die Arbeitsfähigkeit der Mitarbeitenden sicher

Ermöglicht die Bewältigung konkreter technisch-organisatorischer Veränderungen (Geißler und Orthey 1990, aber auch Heuer ); erhöht die „Mobilität“, damit „Mitarbeiter Veränderungen erkennen und diese vorantreiben" (Rodehuth 1999, S. 183), sichert die Innovationsfähigkeit des Unternehmens (Seyda und Werner 2014)

Fördert Auseinandersetzung und Identifizierung mit der sowie Integration in die Unternehmenskultur

Zielt auf den Schutz der gesundheitlichen Arbeitsfähigkeit der Mitarbeiter

Unterstützt die persönliche Entwicklung der Mitarbeitenden, fördert das Lernen von Lernen, ermöglicht die Auseinandersetzung mit der eigenen Biografie, primärer Fokus liegt nicht auf der Arbeitsfähigkeit

\section{Beschreibung}

Erhöht die Attraktivität des Unternehmens für potenzielle Mitarbeitende und Kunden

Stellt sicher, dass gesetzliche Anforderungen und Unternehmensrichtlinien erfüllt werden; auch Zertifizierungen

Belohnt und motiviert Mitarbeitende, bindet sie an das Unternehmen, wertet Berufsgruppen gegenüber anderen auf bzw. ab (Bsp. Pfleger/ Ärzte)

Legitimiert Beförderungsentscheidungen im Unternehmen bzw. dient als Filter (u. a. Schmitz 1980; Diedrich 1988; Quenzler 2008; Heuer 2010), reproduziert soziale Machtverhältnisse im Unternehmenskontext (Dewe und Feistel 2013)

Lenkt von anderen Themen wie Arbeitsverdichtung ab (Perspektive Betriebsrat, daher nicht gleich Motivationsfunktion)

Wertet die Tätigkeit der einzelnen WB-Mitarbeitenden oder der WB-Abteilung innerhalb des Unternehmens auf (induktiv), führt zu einem größeren Mitarbeiterstab für die Personalabteilung (Heuer 2010)

Ermöglicht kollegialen Austausch/Networking, stärkt Zusammenhalt (insbesondere oberste Führungsebene Fall A Programm)
Deduktiv, u. a.

Diedrich 1988

Deduktiv

Deduktiv, u. a. Weber 1985, S. $61 \mathrm{ff}$.

Induktiv

Deduktiv, u. a.

Dewe und Feistel 2013, induktiv erweitert

\section{Quelle}

Deduktiv, alle eingangs genannten Autoren

Induktiv

Deduktiv, alle eingangs genannten Autoren

Deduktiv

Induktiv

Induktiv/deduktiv

Induktiv 
cher Handlungslogik (Orientierungen, Handlungs- und Kommunikationsvoraussetzungen) - der die bWB unterliegt - ist es, das Überleben der Organisation dauerhaft sicherzustellen. Der kommunikative Rahmen der bWB ist die „ständige Frage nach ihrer innerbetrieblichen Existenzberechtigung" (S. 140) und die Frage nach Weiterbildungskosten und -erträgen. In der bWB kommt es daher nicht auf die Teilnehmenden, sondern auf die Organisation an. BWB ist dabei eine austauschbare Möglichkeit, um Anpassungsfähigkeit und Zukunftssicherheit der Organisation und damit die Reproduktion der Betriebsform sicherzustellen.

Phänomenologisch zeigt sich der Charakter der beigeordneten Bildung z. B. am

Zuschnitt von Weiterbildungsentscheidungen, die nicht die gesamte lernende

Person in den Mittelpunkt stellt (...), sondern an notwendigen betrieblichen

Funktionen orientiert sind (Heuer 2010, S. 89).

Die meisten der oben genannten Funktionen verweisen auf die Zielperspektiven von Unternehmen und Mitarbeitenden sowie einige auf die Zielperspektive der WBAbteilung. Auffällig ist, dass so gut wie keine eine gesellschaftliche Zielperspektive aufweist. ${ }^{15}$ Man kann diese Zielperspektiven auch als Begründungszusammenhänge sehen, auf die Programmplanende rekurrieren, um Entscheidungen zu begründen (vgl. auch Pohlmann 2015). Dazu zählen neben pädagogischen Prinzipien (Zielperspektiven Individuen und Gesellschaft) auch ökonomische Interessen (Zielperspektiven Unternehmen und Mitarbeitende) und gesellschaftliche Ansprüche. Für die Auswertung der Daten des hier beschriebenen Projekts haben wir als vierten Begründungszusammenhang mikropolitische Überlegungen (Zielperspektive WBAbteilung) hinzugefügt.

Als methodische Einschränkung ist zu nennen, dass durch die gewählte Erhebungsmethode des Interviews möglicherweise Funktionen, die die Akteure bewusst nicht zur Sprache bringen, nicht berücksichtigt werden. Denkbar ist auch, dass einzelne Akteure bestimmte Funktionen gezielt nicht ansprechen oder überbetonen, weil es ihren eigenen Interessen dient (vgl. zu nicht-kommunizierten Funktionen von Coaching: Taffertshofer 2006, S. 10). So könnte z. B. der Vorstand ein Interesse daran haben, die Legitimationsfunktion nicht zu offenkundig anzusprechen oder die WB-Abteilung die Qualifizierungsfunktion in den Vordergrund stellen, um ihre Arbeit im Unternehmen zu rechtfertigen (Heuer 2010, S. 124 f.). Gleichzeitig ist ein methodischer Vorteil darin zu sehen, dass die Kategorie der zugeschriebenen Funktionen induktiv entwickelt wurde, so dass keine direkten Fragen im Leitfaden hierzu bestehen, die eventuell Rechtfertigungen etc. hervorgerufen hätten.

\subsection{Fallanalyse}

Anhand der Fallanalyse werden die zuvor beschriebenen Zusammenhänge beispielhaft verdeutlicht. Alle Beispiele stammen aus Fall A. Es wird gezeigt, wie mithilfe

\footnotetext{
15 Dabei wäre dies möglich: „Erst in der Perspektive der Rückbindung an das wirtschaftliche Handeln zum Nutzen einer Gesellschaft erhält auch die betriebliche Weiterbildung einen humanistischen Anklang, weil er den Sinn benennt, dem wirtschaftliches Handeln verpflichtet ist“ (Heuer 2010, S. 117).
} 
der zugeschriebenen Funktionen das Zustandekommen eines bestimmten Angebotsspektrums erklärt werden kann.

\subsubsection{Das Unternehmen und die Akteure}

Unternehmen A agiert im Gesundheitsbereich und hat über 5000 Mitarbeitende. Das Unternehmen zeichnet sich durch eine sehr heterogene Mitarbeiterschaft aus: Neben Ärzten und Pflegepersonal, das auch ausgebildet wird, gibt es weitere gesundheitsnahe Berufsgruppen, wie z. B. Hebammen, sowie andere Berufsgruppen, z. B. in der Verwaltung. Mehrfach wird in den Interviews darauf verwiesen, dass sich das Unternehmen in einer Sanierungsphase befindet und das WB-Budget deshalb sehr gering sei. Darüber hinaus wird das Gesundheitswesen als stark gesetzlich reguliert beschrieben. Die nachfolgende Tab. 2 gibt einen Überblick über die Akteurinnen und Akteure, alle Interviewpartner wurden mit * markiert. Die nachfolgenden Ausführungen konzentrieren sich auf die planenden Akteurinnen und Akteure.

\subsubsection{Das WB-Angebot: Fünf verschiedene Programmarten}

Wie bereits allgemein dargestellt, finden sich auch in Unternehmen A verschiedene Programmarten. Zwei Aspekte sind im Vergleich zu den anderen beiden Fällen besonders interessant. Zum einen finden in Unternehmen A mehr Angebote außerhalb des Programmkatalogs statt, v. a. maßgeschneiderte Angebote und Bereichs- bzw. Berufsgruppen-interne Angebote ohne Beteiligung der WB-Abteilung.

Dann haben wir am Standort noch mal ein eigenes Fort- und Weiterbildungsprogramm für den Pflegedienst etabliert, ... (...) Das is' jetzt gerade auf der letzten Stationsleitungskonferenz raus gegeben worden (Pflegeleitung Standort 3).

Zum anderen fällt in Bezug auf den Programmkatalog auf, dass dieser Angebote mehrfach enthält, was zu einem großen Umfang führt. Darüber hinaus sind Angebote wie Yoga enthalten, diese werden von der WB-Leitung als ,goodies“ bezeichnet.

Tab. 2 Akteurinnen und Akteure im Fall A in Anlehnung an das Mehrebenenmodell der Weiterbildung (Schrader 2011)

\begin{tabular}{|c|c|}
\hline Ebene & Akteurinnen und Akteure \\
\hline Lerngelegenheiten & Lernende*, interne* ${ }^{*}$ und externe Dozenten \\
\hline Unternehmensebene & $\begin{array}{l}\text { WB-Leitung*, WB-Team*, Ärzteschaft*, Pflegschaft*, Ge- } \\
\text { schäftliche Leitung*, Verwaltung, Betriebsrat*, Unternehmens- } \\
\text { leitung }\end{array}$ \\
\hline Unmittelbare Umwelt & Wettbewerber \\
\hline $\begin{array}{l}\text { (Bildungs-)Politik auf Landes- und } \\
\text { Bundesebene }\end{array}$ & Ärztekammer, Krankenkassen, Gesetzgeber \\
\hline $\begin{array}{l}\text { Bildungspolitik auf supranationaler } \\
\text { Ebene }\end{array}$ & EU, OECD, UNESCO u. a. \\
\hline
\end{tabular}




\subsubsection{Zugeschriebene Funktionen im Fall A}

Um das Zustandekommen der Programme beispielhaft zu verstehen, werden nun die Funktionen betrachtet, die die verschiedenen Akteurinnen dem Programmkatalog vorrangig zuschreiben. Einige Perspektiven lassen sich durch die beruflichen Hintergründe der Interviewten erklären.

Aus Sicht der WB-Leitung stehen Image- und Akquisefunktion (,Fachkräfte werben“), Compliance-Funktion (Vorgaben erfüllen, um kein Geld zu verlieren) sowie Gratifikations-, Motivations- und Mitarbeiterbildungsfunktion im Vordergrund (Bspw. sind die Angebote im Programmkatalog nach Berufsgruppen sortiert). So kommt es zu der Mehrfachnennung und dem großen Umfang des gedruckten Programmkatalogs - was wiederum von Vorteil für die Image- und Akquisefunktion ist:

- Image- und Akquisefunktion: „Und jetzt erzählt mir zum Beispiel die Chefärztin, wissen Sie, Frau XY, wenn bei mir Bewerber kommen, haben mir jetzt schon drei gesagt, dann nehm 'ich mir Ihr Buch, ja oder Euer Buch, und zeig denen das, was wir alles haben hier" (Leitung WB-Abteilung).

- Gratifikations-, Motivations-, Mitarbeiterbindungsfunktion: „Der Konzern sieht, dass ich da bin. Und hat auch was für mich. Ob ich,s nutze, wie gesagt, bleibt auch wieder was anderes" (Leitung WB-Abteilung).

Anders als bspw. in Unternehmen B (WB-Leitung mit erwachsenenpädagogischem Hintergrund und spezifischer Unternehmensphilosophie) wird die Entwicklungsfunktion nicht in den Vordergrund gerückt. Das lässt sich mit dem beruflichen Hintergrund der WB-Leitung erklären. Sie plädiert dafür, dass es schlauer sei, nicht nur Pädagogen mit Personalentwicklung zu betrauen, sondern Personen wie sie:

dadurch, dass ich aus dem medizinischen Bereich komme, und das Kerngeschäft extrem gut kenne [I: $\mathrm{mhm}]$ weiß ich natürlich unglaublich viel über das, was brauchen die Menschen (Leitung WB-Abteilung).

Demgegenüber weisen andere Akteurinnen und Akteure dem Programmkatalog bzw. bWB allgemein andere Funktionen zu. Dadurch, dass diese teilweise nicht erfüllt werden, entstehen weitere Angebote außerhalb des Programmkatalogs. Als Beispiel ein Zitat der bereits oben zitierten Pflegeleitung, die die Bewältigungs- und Flexibilitätsfunktion in den Vordergrund rückt:

Das hat auch wieder etwas mit der medizinischen Ausrichtung zu tun. Wenn jetzt auf einmal die Geschäftsführung sagt, wir wollen jetzt etwas etablieren, [I1: Mhm.] muss ich mal flächendeckend ganz schnell 950 Mitarbeiter schulen. (...) Deshalb brauch' ich standortbezogene Fort- und Weiterbildungsprogramme (Pflegeleitung Standort 3).

Hier wird auch deutlich, dass hinter einer Funktion verschiedene Ziele stehen können, für das obige Zitat z. B. Wettbewerbsvorteil und Arbeitsqualität.

Ein weiteres Zitat weist auf die Perspektive der Ärzteschaft hin. Für den Chefarzt sollte Weiterbildung v. a. eine Qualifizierungsfunktion erfüllen - er schreibt diese 
Funktion dem Programmkatalog allerdings in geringerem Maße zu. Dementsprechend wünscht er sich einen höheren Anteil des WB-Budgets für seine Abteilung:

Ja, es gibt, es gibt so'n allgemeines Weiterbildungsbudget, was außerordentlich knapp [I: Mhm.] is'. Ähm, wobei 'n Teil, 'n großen Teil des Budgets, ehrlicherweise muss man natürlich sagen, is' in dieser Personalentwicklung verbraten. (...) Mh und dann ähm gibt es äh für die Abteilungen selbst ein sehr kleines Budget (Chefarzt).

Mit Merton (1967) lassen sich auch latente, also unerwartete und unerkannte, Funktionen benennen. ${ }^{16}$ Dies wird z. B. in der Interpretation der WB-Angebote durch ein Betriebsratsmitglied, das dem Programmkatalog auch eine Ablenkungsfunktion zuschreibt, deutlich (vom beruflichen Hintergrund ebenso Arzt):

es ist ein netter Versuch (...) auf die Leute zuzugehen [Ew: ja.] und ihnen (...) Möglichkeiten zu schaffen, äh mit dem Stress irgendwie umzugehen, aber es, es reicht nicht. Es sind, es sind - ich sag' jetzt mal Ersatzbefriedigungen. (...) Weil im Grunde genommen, an dem Grundproblem, das was die Menschen eigentlich belastet, wird gar nichts gemacht, sondern es wird nur an den Symptomen rumgedoktert [I1: Mhm.] und nicht die, die Ursache oder die Krankheit beseitigt. [I1: Mhm.] Und die Krankheit ist ganz einfach Arbeitsverdichtung (Betriebsrat).

\subsubsection{Handlungskoordination}

In den Zitaten der verschiedenen Akteurinnen und Akteure wird deutlich, dass diese sehr unterschiedliche Interessen verfolgen. Der WB-Leitung geht es z. B. stark um die Profilierung ihrer Abteilung im Unternehmen; auf der Ebene der Standorte geht es darum, sich im Wettbewerb gegen andere Krankenhäuser zu positionieren; dem Chefarzt geht es darum, WB als Statussymbol der Ärzteschaft weiter nutzen zu können, während die Pflegschaft durch mehr WB auf ein höheres Ansehen hofft und der Betriebsrat das Ziel niedrigerer Arbeitsverdichtung verfolgt.

Handlungskoordination meint also nicht nur inhaltliche und konzeptionelle Abstimmungsprozesse (Gieseke 2003), sondern auch das Aushandeln von Macht und Interessen über die Zuschreibung von Funktionen an bWB.

Handlungskoordination findet in Fall A teilweise in jährlichen Planungsgesprächen, z. B. zwischen WB-Abteilung und Pflegedirektorenkonferenz oder zwischen WB-Abteilung und Geschäftsführern der verschiedenen Standorte, sowie in kurzfristigen Gesprächen, z. B. zwischen Führungskräften und der WB-Abteilung statt. Gleichzeitig werden grundlegende Entscheidungen wie z. B. die Aufteilung des Budgets hauptsächlich von der WB-Abteilung bzw. explizit der WB-Leitung getroffen.

Als Beispiel soll die Verteilung des Budgets für bWB betrachtet werden. Diese wird wie bereits beschrieben von der WB-Leitung vorgenommen. Sie entscheidet, wie viel Budget für den Programmkatalog verwendet wird, wie viel Budget dabei welcher Berufsgruppe zugutekommt und wieviel Budget darüber hinaus für externe,

16 Vgl. auch zu den latenten Funktionen von Coaching Kühl 2008; Taffertshofer 2006. 
maßgeschneiderte und bereichsinterne Angebote an einzelne Standorte und Abteilungen gegeben wird.

Frau XY versucht das [Budget] schon gerecht zu verteilen. [I: Ja] Prozentual gemessen an der, äh an der Kopfzahl der der der Pflege also der der Berufsgruppen, [I: Mhm] immer. Größe der Berufsgruppe. [I: Okay. Ja] Ne, da muss man ja, das schon gerecht verteilen (Mitarbeiter WB-Abteilung).

Der Modus der Handlungskoordination der anderen Akteurinnen und Akteure ist somit zunächst die Beobachtung (vgl. Lange und Schimank 2004, S. 19 ff.). Gleichzeitig kann die oben zitierte Aussage des Chefarztes, ein Großteil des Budgets ,wird verbraten“, als Beeinflussung verstanden werden. Es stellt eine direkte Bedrohung für das Interesse der WB-Abteilung, sich intern zu profilieren, dar. Die Planungssituation ist somit durch eine nicht-konsensuale Interessenkonstellation und eine asymmetrische Machtkonstellation gekennzeichnet (Cervero und Wilson 1994).

Dieser Konflikt setzt sich dann an den einzelnen Standorten fort. Dort entscheiden Geschäftsführung, Ärztliche Direktion und Pflegedirektion, wie das standortbezogene Budget für WB verwendet wird, hier findet sich der Modus der Verhandlung.

also Geschäftsführende Direktorin, Ärztlicher Direktor, die arbeiten da schon ganz eng. Ich bin in alle Prozesse mit involviert, da plant dann jeder aufgrund, also äh anhand seiner Berufsgruppe, die er vertritt. Das ist nicht immer reibungslos und äh schon alleine bei der Zielfindung is' ganz klar, dass das 'n Prozess is'. Das wird ausdiskutiert und dann äh gibt 's natürlich 'n Budget und das is' immer zu gering, ganz klar. Aber dann muss man fighten. Also typische Argumente sind natürlich äh, äh für, für die Mediziner: (...) unsere Fort- und Weiterbildungsmaßnahmen sind aber wesentlich teurer als die für Pflegekräfte. Is' auch so auf 'm Markt. Alles ganz klar. Da sag' ich aber wieder auf der Gegenseite: Ich hab' aber die größte Berufsgruppe. Ich muss mehr Mitarbeiter schulen. Also, also das, das ergänzt sich (Pflegedirektion).

\section{Diskussion und Ausblick}

Das vorgestellte Forschungsvorhaben zielte auf einen empirischen Beitrag zur Theoriebildung zur Programmplanung in der bWB. Als wichtigste Erträge sind zu nennen: Die detaillierte Darstellung der beteiligten Akteurinnen und Akteure und ihrer Interessen, die empirisch fundierte Ausdifferenzierung und Kategorisierung der zugeschriebenen Funktionen bWB unter Berücksichtigung der Akteursperspektive, die Beschreibung der verschiedenen Programmarten (Fragestellung 1) und das Aufzeigen der Zusammenhänge von zugeschriebenen Funktionen, Planungsprozessen und Programmarten in einer Grounded Theory (Fragestellung 2). Angleichungshandeln in der bWB kann als spezifischer Auslegungsprozess von Bildung mit den zugeschriebenen Funktionen beschrieben werden. Es kann hiermit die Diversität der Programmentwicklung durch die empirische Erschließung der Zuschreibungen der Akteure beschrieben werden. 
Es handelt sich um eine empirische Detailanalyse zur Differenzierung der Programmplanung bWB, wie sie beispielsweise mit Erhebungen wie CVTS nicht nachgezeichnet werden kann, die Studien ergänzen sich vielmehr komplementär.

Nicht im Fokus der Untersuchung standen die Teilnehmenden als Akteure mit ihren Nutzenerwartungen (vgl. Fleige 2015), die aber natürlich in einem Mehrebenensystem dazugehören. Hier lag der Fokus auf der Planung, somit auf der Mesoebene. In Bezug auf die Teilnehmenden und Planungspartizipation ist weitere Forschung notwendig. In Bezug auf die Akteurinnen und Akteure kann darüber hinaus auch das Zusammenspiel von individuellen und kollektiven Einflussfaktoren auf die zugeschriebenen Funktionen ein interessanter Ausgangspunkt für weitere Forschungsarbeiten sein. Der Zugang zu den Interviewpartnern über die Leitung der WB-Abteilung ist als methodische Limitation $\mathrm{zu}$ sehen. Anschlussforschung könnte sich gezielt mit Nicht-Akteuren auseinandersetzen und z. B. den Weg einer teilnehmenden Beobachtung wählen.

Unsere Analyse ist in der Programmforschung verortet; weitere Anschlussmöglichkeiten liegen in der soziologischen Rahmenanalyse sowie der pädagogischen Organisationsforschung. Die Zuschreibung von Funktionen könnte rahmenanalytisch (Was geht hier eigentlich vor?) (vgl. Goffman 1980) untersucht werden. Funktionen können von den Akteuren je nach Rahmen (Interpretationsschemata/ Orientierungsmuster (vgl. Schäfer 2015, S. 235 ff.)) unterschiedlich zugewiesen werden. Ein Rahmen kann z. B. die jährliche Planungssitzung der WB-Abteilung, die Bedarfserhebung an einem Standort etc. sein, das Geschehen kann jedoch durch Rahmungsprozesse der Individuen unterschiedlich gerahmt werden.

Interessant könnte es ebenfalls perspektivisch sein, Kommunikationsprozesse während des Planungshandelns in der bWB als eingebettet in Kontexturen zu untersuchen. Kontexturen meinen gesellschaftliche Erwartungshorizonte, Orientierungsschemata, „semantisch-kommunikative Engführungen“ (Vogd 2009, S. 31), die unterschiedliche Funktionssysteme und Logiken abbilden. Mittels Kontexturanalyse als Erweiterung der dokumentarischen Methode für die dokumentarische Organisationsforschung (vgl. Jansen et al. 2015) ließe sich untersuchen, wie in der betrieblichen Weiterbildungsplanung unterschiedliche Logiken gleichzeitig bestehen (polykontexturale Verhältnisse).

Für die Praxis bWB ist das Sichtbarmachen der verschiedenen Funktionen und den sich darin spiegelnden Akteursinteressen eine wichtige Grundlage für eine macht- und interessenkritisch reflektierte und damit professionelle Programmplanung. Nur wer die zugeschriebenen Funktionen der anderen Akteurinnen und Akteure kennt, kann daran anknüpfen und seine eigenen vertreten - auch erwachsenenpädagogisch begründete Funktionen mit Fokus auf Teilhabe, Bildung und Entwicklung von Individuen. Gleichzeitig ist in der Kombination der Ergebnisse mit den CVTS-Daten von einer professionell-reflektierten Programmplanung auch ein positiver Einfluss auf eine hohe WB-Beteiligung zu erwarten (vgl. Käpplinger und Lichte 2012). Vertiefende Analysen zu den beruflichen Hintergründen, insbesondere auch zu erwachsenenpädagogischen Kompetenzen der Planenden - d. h. zu ihrer erwachsenenpädagogischen Professionalität - und einem Einfluss auf die zugeschriebenen Funktionen, erscheinen vielversprechend. Die Kategorie der zugeschriebenen Funk- 
tionen ist darüber hinaus in ihrer Übertragbarkeit auf andere Bereiche beigeordneter Bildung außerhalb der bWB zu prüfen und möglicherweise zu ergänzen.

Open Access. Dieser Artikel wird unter der Creative Commons Namensnennung 4.0 International Lizenz (http://creativecommons.org/licenses/by/4.0/deed.de) veröffentlicht, welche die uneingeschränkte Nutzung, Verbreitung und Wiedergabe für beliebige Zwecke erlaubt, sofern Sie den/die ursprünglichen Autor(en) und die Quelle ordnungsgemäß nennen, einen Link zur Creative Commons Lizenz beifügen und angeben, ob Änderungen vorgenommen wurden.

\section{Literatur}

Altrichter, H., \& Heinrich, M. (2007). Kategorien der Governance-Analyse und Transformationen der Systemsteuerung in Österreich. In H. T. B. Altrichter, \& J. Wissinger (Hrsg.), Educational Governance. Handlungskoordination und Steuerung im Bildungssystem (S. 55-103). Wiesbaden: VS Verlag für Sozialwissenschaften.

Altrichter, H., Brüsemeister, T., \& Wissinger, J. (Hrsg.). (2007). Educational Governance. Handlungskoordination und Steuerung im Bildungssystem. Wiesbaden: VS Verlag für Sozialwissenschaften.

Bäumer, J. (1999). Weiterbildungsmanagement. Eine empirische Analyse deutscher Unternehmen. Bd. 1. München: Hampp.

Becker, M. (2011). Systematische Personalentwicklung - Planung, Steuerung und Kontrolle im Funktionszyklus (2. Aufl.). Stuttgart: Schäffer Poeschel.

Behrmann, D. (2010). Lernen in der Organisation. Rekonstruktionen zum Verhältnis von individuellem und organisationalem Lernen. In C. Heidsiek \& H. Geißler (Hrsg.), Organisationslernen im 21. Jahrhundert. Festschrift für Harald Geißler (S. 93-103). Frankfurt a. M. u.a.: Lang.

Blumer, H. (1972). Der methodologische Standort des symbolischen Interaktionismus. In Alltagswissen und Interaktion (S. 80-101). Reinbek: Rowohlt.

BMBF - Bundesministerium für Bildung und Forschung (2014). Weiterbildungsverhalten in Deutschland. AES 2014 Trendbericht. https://www.bmbf.de/pub/Weiterbildungsverhalten_in_Deutschland_ 2014.pdf. Zugegriffen: 3. Nov. 2015.

Bogner, A., \& Menz, W. (2002). Expertenwissen und Forschungspraxis: die modernisierungstheoretische und die methodische Debatte um die Experten. Zur Einführung in ein unübersichtliches Problemfeld. In A. Bogner, B. Littig, \& W. Menz (Hrsg.), Das Experteninterview. Theorie, Methode, Anwendung (S. 7-30). Opladen: Leske und Budrich.

Cervero, R. M., \& Wilson, A.L. (1994). Planning responsibly for adult education. A guide to negotiating power and interests. San Francisco: Jossey-Bass.

Dewe, B., \& Feistel, K. (2013). Betriebliche Weiterbildung. Materialien in didaktischer und bildungsökonomischer Perspektive. Stuttgart: Steiner.

Diedrich, A. (1988). Effizienz betrieblicher Weiterbildung: Betriebliche Weiterbildung als einzelwirtschaftliches Entscheidungsproblem. Köln: Wirtschaftsverlag Bachem.

Dobischat, R., \& Düsseldorff, K. (2010). Personalentwicklung und Arbeitnehmer. In R. Tippelt, \& A. von Hippel (Hrsg.), Handbuch Erwachsenenbildung/Weiterbildung (S. 917-938). Wiesbaden: VS Verlag für Sozialwissenschaften.

Dollhausen, K. (2008). Planungskulturen in der Weiterbildung: Angebotsplanung zwischen wirtschaftlichen Erfordernissen und pädagogischem Anspruch. Bielefeld: W. Bertelsmann.

Faulstich, P. (1998). Strategien der betrieblichen Weiterbildung. Kompetenz und Organisation. München: Vahlen.

Fleige, M. (2015). Nutzenvorstellungen von Teilnehmenden in beruflichen und erwerbsbiographischen Übergangssituationen. Befunde aus zwei Untersuchungen. In S. Schmidt-Lauff, H. v Felden, \& H. Pätzold (Hrsg.), Transitionen in der Erwachsenenbildung. Gesellschaftliche, institutionelle und individuelle Übergänge. Dokumentation der Jahrestagung der Sektion Erwachsenenbildung der Deutschen Gesellschaft für Erziehungswissenschaft 2014 (S. 189-200). Opladen, Berlin, Toronto: Budrich.

Flick, U. (2004). Triangulation - Eine Einführung. Wiesbaden: VS Verlag für Sozialwissenschaften.

Geißler, K. A., \& Orthey, F. M. (1990). Lernen in Unternehmen als Modernisierungsstrategie. Situation und Perspektiven betrieblicher Bildungspolitik. In K. A. Geißler, A. Orthey, \& S. Laske (Hrsg.), Handbuch Personalentwicklung. Köln: Deutscher Wirtschaftsdienst. 
Gieseke, W. (2003). Programmplanungshandeln als Angleichungshandeln. In W. Gieseke (Hrsg.), Institutionelle Innensichten der Weiterbildung (S. 189-211). Bielefeld: W. Bertelsmann.

Gieseke, W. (2007). Das Forschungsarrangement Perspektivverschränkung. In W. Gieseke, \& O. Schäffter (Hrsg.), Qualitative Forschungsverfahren in Perspektivverschränkung. Dokumentation des Kolloquiums anlässlich des 60. Geburtstages von Frau Prof. Dr. Wiltrud Gieseke am 29. Juni 2007, Erwachsenenpädagogischer Report (Bd. 11, S. 10-22). Berlin: Humboldt-Universität.

Gieseke, W., \& Heuer, U. (2011). Weiterbildungsentscheidungen und beigeordnete Bildung. In R. Arnold, \& A. Pachner (Hrsg.), Lernen im Lebenslauf (S. 107-127). Baltmannsweiler: Schneider.

Goffman, E. (1980). Rahmen-Analyse. Ein Versuch über die Organisation von Alltagserfahrungen. Frankfurt a. M.: Suhrkamp.

Habeck, S. A. (2015). Freiwilligenmanagement. Exploration eines erwachsenenpädagogischen Berufsfeldes. Wiesbaden: Springer.

Harney, K. (1998). Handlungslogik betrieblicher Weiterbildung. Stuttgart: Hirzel.

Heuer, U. (2010). Betriebliche Weiterbildungsentscheidungen: Aushandlungsprozesse und Bildungscontrolling. Fallstudienbericht. http://www.bibb.de/veroeffentlichungen/de/publication/download/id/ 6221. Zugegriffen: 3. Nov. 2015.

Hippel, A. von (2011). Programmplanungshandeln im Spannungsfeld heterogener Erwartungen: ein Ansatz zur Differenzierung von Widerspruchskonstellationen und professionellen Antinomien. ReportZeitschrift für Weiterbildungsforschung, 1, 45-57.

Hippel, A. von (2012). Programmplanung als professionelles Handeln - „Angleichungshandeln“ und „Aneignungsmodi“ im aktuellen Diskurs der Programm- und Professionsforschung. In B. Käpplinger, \& S. Schmidt-Lauff (Hrsg.), Engagement für die Erwachsenenbildung. Ethische Bezugnahmen und demokratische Verantwortung (S. 131-143). Wiesbaden: VS Verlag für Sozialwissenschaften.

Hippel, A. von (i.E.): Programmplanungskulturen in der betrieblichen Weiterbildung - Eine Programmanalyse zu Funktionen betrieblicher Weiterbildungsprogramme. Tagungsband der Jahrestagung Sektion Erwachsenenbildung der DGfE.

Jansen, T., Schlippe, A. von, \& Vogd, W. (2015). Kontexturanalyse - ein Vorschlag für rekonstruktive Sozialforschung in organisationalen Zusammenhängen. Forum Qualitative Sozialforschung, 16(1), 4.

Käpplinger, B. (2009). Betriebliche Weiterbildungsentscheidungen: Aushandlungsprozesse und Bildungscontrolling. Abschlussbericht. http://www2.bibb.de/bibbtools/tools/fodb/data/documents/pdf/eb_ 22203.pdf. Zugegriffen: 3. Nov. 2015.

Käpplinger, B. (2012). Auf die Unterschiede kommt es an. Bildungscontrolling. Weiterbildung, 1, 12-15.

Käpplinger, B., \& Lichte, N. (2012). Erhöhung der Weiterbildungsbeteiligung durch professionelles Weiterbildungspersonal. WSI-Mitteilungen: Zeitschrift des Wirtschafts- und Sozialwissenschaftlichen Instituts in der Hans-Böckler-Stiftung, 65, 347-381.

Käpplinger, B. (i.E.) Theorien und Theoreme der betrieblichen Weiterbildung. In R. Tippelt, \& A. von Hippel (Hrsg.), Handbuch Erwachsenenbildung/Weiterbildung 6. Aufl. Wiesbaden: VS Verlag für Sozialwissenschaften.

Kühl, S. (2008). Coaching und Supervision. Zur personenorientierten Beratung in Organisationen. Wiesbaden: VS Verlag für Sozialwissenschaften.

Lange, S., \& Schimank, U. (2004). Governance und gesellschaftliche Integration. Reihe Governance (Bd. 2), Wiesbaden: VS Verlag für Sozialwissenschaften.

Mayring, P. (2003). Qualitative Inhaltsanalyse. Grundlagen und Techniken. Weinheim: Beltz.

McLean, S. (2000). „Between rationality and politics“: Autobiographical portraits of adult education programme planning. International Journal of Lifelong Education, 19(6), 493-505.

Merkens, H. (1997). Stichproben bei qualitativen Studien. In B. Friebertshäuser, \& A. Prengel (Hrsg.), Handbuch Qualitative Forschungsmethoden in der Erziehungswissenschaft (S. 97-106). Weinheim: Juventa.

Merton, R. K. (1967). Funktionale Analyse. In H. Hartmann (Hrsg.), Moderne amerikanische Soziologie (S. 169-214). Stuttgart: Enke.

Neuberger, O. (1994). Personalentwicklung (2. Aufl.). Stuttgart: Lucius \& Lucius.

Nienhüser, W., \& Rodehuth, M. (1995). Technischer Wandel und betriebliche Bildungsentscheidungen. Grundlagen der Weiterbildung, 6, 110-113.

Nolda, S. (2009). Programmanalyse - Methoden und Forschungen. In R. Tippelt, \& A. von Hippel (Hrsg.), Handbuch Erwachsenenbildung/Weiterbildung (3. Aufl. S. 293-307). Wiesbaden: VS Verlag für Sozialwissenschaften.

Pohlmann, C. (2015). Angebotsplanung von Bildungsurlaubsveranstaltungen im Spannungsfeld gesellschaftlicher Ansprüche, erwachsenenpädagogischer Ansprüche, erwachsenenpädagogischer Prinzipien und ökonomischer Interessen. In S. Robak, H. Rippien, L. Heidemann, \& C. Pohlmann (Hrsg.), 
Bildungsurlaub - Planung, Programm und Partizipation. Eine Studie in Perspektivverschränkung (S. 69-112).

Pullig, K.-K. (1991). Weiterbildungskonzepte deutscher Unternehmen - eine Analyse von Weiterbildungsprogrammen. Zeitschrift für Personalforschung, 3, 219-237.

Quenzler, A. (2008). Der Nutzen betrieblicher Weiterbildung für Großunternehmen: eine empirische Analyse unter bildungsökonomischen Aspekten am Beispiel der innerbetrieblichen Führungskräfteentwicklung der Audi AG. Göttingen: Cuvillier.

Robak, S. (2012). Programmanalysen: Einführung in die Erstellung von Codesystemen. http://www.diebonn.de/Institut/Dienstleistungen/Servicestellen/Programmforschung/Methodische_Handreichungen/ codiersysteme/Programmanalyse-Codesysteme-Robak.pdf. Zugegriffen: 3. Nov. 2015.

Robak, S. et al. (2015). Bildungsurlaub - Planung, Programm und Partizipation. Eine Studie zur Perspektivverschränkung. Frankfurt a. M.: Lang.

Rodehuth, M. (1999). Weiterbildung und Personalstrategien. Eine ökonomisch fundierte Analyse der Bestimmungsfaktoren und Wirkungszusammenhänge. München: Hampp.

Schäfer, H. W. (2015). Identität als Netzwerk. Habitus, Sozialstruktur und religiöse Mobilisierung. Wiesbaden: Springer.

Schiersmann, C., Iller, C., \& Remmele, H. (2001). Aktuelle Ergebnisse zur betrieblichen Weiterbildungsforschung. Report. Literatur- und Forschungsreport Weiterbildung, 48, 8-36.

Schlutz, E. (2006). Bildungsdienstleistungen und Angebotsentwicklung. Münster: Waxmann.

Schmitz, E. (1978). Leistung und Loyalität - Berufliche Weiterbildung und Personalpolitik in Industrieunternehmen. Stuttgart: Klett-Cotta.

Schmitz, E. (1980). Betriebliche Weiterbildung als Personalpolitik. In A. Weymann (Hrsg.), Handbuch für die Soziologie der Weiterbildung (S. 120-136). Darmstadt: Luchterhand.

Schönfeld, G., \& Behringer, F. (2013). Betriebliche Weiterbildung in Deutschland im europäischen Vergleich. Ergebnisse der dritten europäischen Erhebung zur betrieblichen Weiterbildung (CVTS3). In Bundesinstitut für Berufsbildung (Hrsg.), Wissenschaftliche Diskussionspapiere, Heft 115.

Schrader, J. (2001). Bindung, Vertrag, Vertrauen: Grundlagen der Zusammenarbeit in Weiterbildungseinrichtungen. Hessische Blätter für Volksbildung, 51, 142-154.

Schrader, J. (2011). Struktur und Wandel der Weiterbildung. Bielefeld: W. Bertelsmann.

Seyda, S., \& Werner, D. (2014). IW-Trends. Vierteljahresschrift zur empirischen Wirtschaftsforschung aus dem Institut der deutschen Wirtschaft, 41(4), 1-15.

Stender, J. (2009). Betriebliches Weiterbildungsmanagement. Stuttgart: Hirzel.

Strauss, A., \& Corbin, J. (1996). Grounded Theory: Grundlagen Qualitativer Sozialforschung. Weinheim: Beltz.

Taffertshofer, A. (2006). Funktionen von Coaching in Organisationen. http://www.uni-bielefeld.de/soz/ forschung/orgsoz/Stefan_Kuehl/pdf/Paper5.2006-Funktionen-von-Coachimg-in-Organisationen. pdf. Zugegriffen: 9. Mai 2016.

Vogd, W. (2009). Rekonstruktive Organisationsforschung. Qualitative Methodologie und theoretische Integration - eine Einführung. Opladen, Berlin, Toronto: Budrich.

Weber, W. (1985). Betriebliche Weiterbildung. Empirische Analyse betrieblicher und individueller Entscheidungen über Weiterbildung. Stuttgart: Poeschel.

Weber, W., Mayrhofer, W., Nienhäuser, W., Rodenuth, M., \& Rüther, B. (1994). Betriebliche Bildungsentscheidungen: Entscheidungsverläufe und Entscheidungsergebnisse. München: Hampp.

Witzel, A. (2000). Das problemzentrierte Interview. Forum qualitative Sozialforschung, 1(1). http://www. qualitative-research.net/index.php/fqs/article/view/\%201132/2519. Zugegriffen: 15. Aug. 2013.

Yin, R.K. (2009). Case Study Research: Design and Methods. London. 